\title{
On the Time Evolution of Limited-Area Ensemble Variance: Case Studies with the Convection-Permitting Ensemble COSMO-E
}

\author{
CHRISTINA KLASA \\ Institute for Atmospheric and Climate Science, ETH Zürich, Zurich, Switzerland \\ MARCo ARPAgAus AND ANDRÉ WALSER \\ Federal Office of Meteorology and Climatology MeteoSwiss, Zurich, Switzerland \\ HEINI WERNLI \\ Institute for Atmospheric and Climate Science, ETH Zürich, Zurich, Switzerland
}

(Manuscript received 11 January 2018, in final form 19 October 2018)

\begin{abstract}
Dynamical processes determining the time evolution of difference kinetic energy (DKE) in a limited-area domain are investigated with the convection-permitting ensemble model COSMO-E for a forecasting period of 4 days. DKE is quantified by means of ensemble variance of the irrotational and nondivergent horizontal wind. For three case studies characterized by contrasting predictability levels of precipitation, it is shown that DKE of the irrotational wind strongly increases during periods of solar-forced moist convective activity and decreases when the latter ceases. The response of DKE of the nondivergent wind is also clearly related to the convective activity, but delayed by a few hours, pointing to interactions between both wind components. Apart from the impact of moist convection, DKE of the nondivergent wind is primarily governed by large-scale advection, imposed at the lateral domain boundaries of the limited-area ensemble. This forcing may also sustain or increase DKE of the irrotational wind when moist convection is absent. Consequently, the large-scale flow and diurnal solar forcing, associated with higher spatiotemporal predictability, determines the overall evolution of the limited-area ensemble variance of the horizontal wind, which increases in the presence of moist convective activity or strong synoptic-scale forcing, and stagnates or decreases otherwise, rendering forecasts of convectionpermitting ensembles valuable beyond the very short forecast range.
\end{abstract}

\section{Introduction}

Numerical weather prediction (NWP) builds on the time integration of the system of equations describing atmospheric motions. Due to the nonlinearity of the equations, small modifications of the initial conditions may grow quickly, limiting the predictability of weather. In the middle of the twentieth century, Edward Lorenz studied predictability limitations of a nonlinear system of equations in an idealized turbulence model (Lorenz 1969). Phase-space trajectories on the Lorenz attractor, which differ by slightly modified initial conditions, may remain close together or diverge strongly, depending on the location of the set of initial conditions on the attractor.

Lorenz applied these findings to atmospheric flows and inferred that atmospheric predictability is scale

Corresponding author: Christina Klasa, christina.klasa@env.ethz.ch dependent. He estimated predictability to be limited to a few weeks for large-scale flows and to only an hour for cumulus-scale motions (Lorenz 1969). These theoretical (or intrinsic) predictability limits are quantified by means of the divergence of model trajectories integrated from slightly varying initial conditions and place an upper bound to the predictability of weather forecasts. In cases of low intrinsic predictability, which is determined by the flow conditions, forecast performance can hardly be improved by more accurate initial conditions (Melhauser and Zhang 2012). In practice, forecast integrations are verified by observations or a model analysis, which also includes predictability limitations due to model deficiencies. Practical predictability thus places a lower bound on forecast quality (Lorenz 1982; Murphy 1988) and can be enhanced when applying more accurate initial conditions and improved model formulations (Zhang et al. 2006; Melhauser and Zhang 2012). These concepts of practical versus 
intrinsic predictability are nicely illustrated in Nielsen and Schumacher (2016, their Fig. 14).

Durran and Gingrich (2014) and Durran and Weyn (2016) revisited error propagation in the Lorenz model and found that errors introduced at the largest scale quickly project onto the smallest scale. The subsequent upscale error growth is indistinguishable from that of errors introduced at the smallest scale after roughly $3 \mathrm{~h}$. In less idealized models, regions of moist convective instability are crucial to the upscale growth of smallscale disturbances (Zhang et al. 2002, 2003; Tan et al. 2004; Walser et al. 2004; Hohenegger et al. 2006; Zhang et al. 2006, 2007; Bei and Zhang 2007; Sun and Zhang 2016), yet sustained error growth only occurs when the perturbation energy can propagate against the mean flow, that is, when the perturbations can continue to grow and are not swept away from the region of instability (Hohenegger et al. 2006). Even small perturbations in one single vertical column located in a convectively unstable region are sufficient to induce error growth comparable to that resulting from introducing the perturbations in the entire model domain (Zhang et al. 2003). This rapid error growth in convectively unstable regions constitutes the first stage in the conceptual error growth model of Zhang et al. (2007). The errors may be dispersed from the area of moist convection by smaller-scale gravity waves, which potentially trigger rapid error growth due to convective instability in the far field (Hohenegger and Schär 2007). Small-scale errors are saturated when convective cells are completely displaced. The small-scale modifications project onto the upper-level flow in the outflow region of the convective cells and induce differences in the precipitation field and the surface pressure distribution. Hence, the errors affect not only increasingly larger geographical areas but also successively larger scales while undergoing geostrophic adjustment (Zhang et al. 2002); that is, the error fields of wind and pressure become geostrophically balanced, which is the transition stage in the error growth model of Zhang et al. (2007). In the final stage, the error grows slowly at large scales due to background baroclinic instability. Zhang et al. (2007) showed that moist processes are not only important in initiating error growth, but also in maintaining subsequent error growth dynamics, in particular at the small scales $(<100 \mathrm{~km})$, and to a lesser extent also at larger scales. Selz and Craig (2015) identified the three conceptual stages of error growth also in real-case simulations, with error saturation beginning at the smallest scales and proceeding upscale. However, the error amplitude may also increase at all scales rather than being transferred in an upscale cascade (Weyn and Durran 2017).
Ensemble variance of nonidealized, limited-area ensemble prediction systems exhibits, during periods of moist convection, a solar-forced diurnal cycle, which is superimposed on the steadily increasing ensemble variance (Nielsen and Schumacher 2016). When moist convective activity vanishes, ensemble variance decreases (Nielsen and Schumacher 2016), in agreement with the idealized study by Zhang et al. (2007). However, in case the synoptic or mesoscale forcing continuously triggers moist convective activity, ensemble variance is augmented further (Nielsen and Schumacher 2016). Thus, strong convective-synoptic scale interactions promote significant and sustained variance growth, which may override the diurnal cycle. Under such conditions, the contribution of convective-scale processes to the total ensemble variance can be as large as the contribution of the synoptic-scale flow. This implies that convective-scale processes prescribe the time evolution of ensemble variance, while interactions with the large-scale flow determine its magnitude (Nielsen and Schumacher 2016).

In this study, we investigate mechanisms that determine the evolution of ensemble variance of the horizontal wind for multiday forecast integrations of an operational ensemble prediction system. The horizontal wind is decomposed into its irrotational and nondivergent components, motivated by their contrasting dynamical implications (see below). Our study addresses the following main research questions:

- Which mechanisms control the overall time evolution of ensemble variance?

- What are the characteristics of ensemble variance under moist convective conditions (i.e., when the predictability level of precipitation is low)?

- How far does the time evolution of the ensemble variance differ when the synoptic-scale forcing of precipitation is strong?

To address these questions, 4-day forecasts of the convection-permitting Consortium for Small-Scale Modeling (COSMO) ensemble prediction system (COSMO-E), which has been operational at MeteoSwiss since May 2016, are investigated for three case studies affecting the area of Switzerland. The cases differ in terms of their predictability level of precipitation and encompass convective summertime precipitation, frontal precipitation, and prefrontal thunderstorm activity. Ensemble variance is quantified by means of the difference kinetic energy (DKE) of the irrotational and nondivergent winds, respectively. Section 2 introduces our methods. The results are presented in section 3 and summarized and discussed in section 4. 


\section{Methods and data}

\section{a. The ensemble prediction system}

COSMO-E is an ensemble prediction system for the region of the European Alps based on the COSMO model, version 5.0. The horizontal grid spacing of $0.02^{\circ}(\approx 2.2 \mathrm{~km})$ allows the nonhydrostatic model with 60 vertical levels to be run in a convection-permitting configuration (Baldauf et al. 2011). The extent of the domain centered over Switzerland (Fig. 1) roughly equals $1280 \mathrm{~km} \times 860 \mathrm{~km}$. The 21 -member ensemble forecasts are run in single precision on graphicsprocessing units at the Swiss National Supercomputing Center [Centro Svizzero di Calcolo Scientifico (CSCS); Fuhrer et al. (2014)]. The initial atmospheric state and the lateral boundary conditions, updated every $3 \mathrm{~h}$, are directly downscaled from the control and the first 20 perturbed members of the global, convection-parameterizing ensemble of the European Centre for Medium-Range Weather Forecasts (ECMWF; www.ecmwf.int). In contrast, the initial surface and soil state is set up in a deterministic manner. Surface and soil variables of all members are initialized with a high-resolution COSMO nudging analysis calculated on the same grid and with the identical external parameters as COSMO-E to use an initial state that fits to the soil model of COSMO and the COSMO-E orography. More details of the model setup, in particular the model physics, are described in Klasa et al. (2018).

The degree to which model physics contribute to the overall forecast uncertainty depends on the synoptic conditions (Gebhardt et al. 2011; Fresnay et al. 2012; Hally et al. 2014; Keil et al. 2014; Kühnlein et al. 2014; Luo and Chen 2015). This is assessed in this study by performing, for each case study, two experiments with and without the stochastically perturbed parameterization tendencies scheme (SPPT; Buizza et al. 1999). With SPPT, total physics tendencies of zonal and meridional winds, temperature, and all moisture-related variables are equally perturbed, with the random numbers drawn from a Gaussian distribution of zero mean and a standard deviation of unity. The range of the random numbers is limited to \pm 0.9 . Spatial and temporal correlation length scales of the random number field are $5^{\circ}$ and $6 \mathrm{~h}$, respectively. The random number field is constant in the vertical, except for the uppermost levels, where a tapering function linearly decreases the impact of the scheme to zero.

\section{b. Decomposition of the horizontal wind}

Helmholtz stated that a vector field $\mathbf{u}$ can be decomposed into an irrotational vector field and a nondivergent vector field. Following Lynch $(1988,1989)$, a horizontal wind field $\mathbf{u}$ may thus be defined as

$$
\mathbf{u}=\nabla \chi+\mathbf{k} \times \nabla \psi
$$

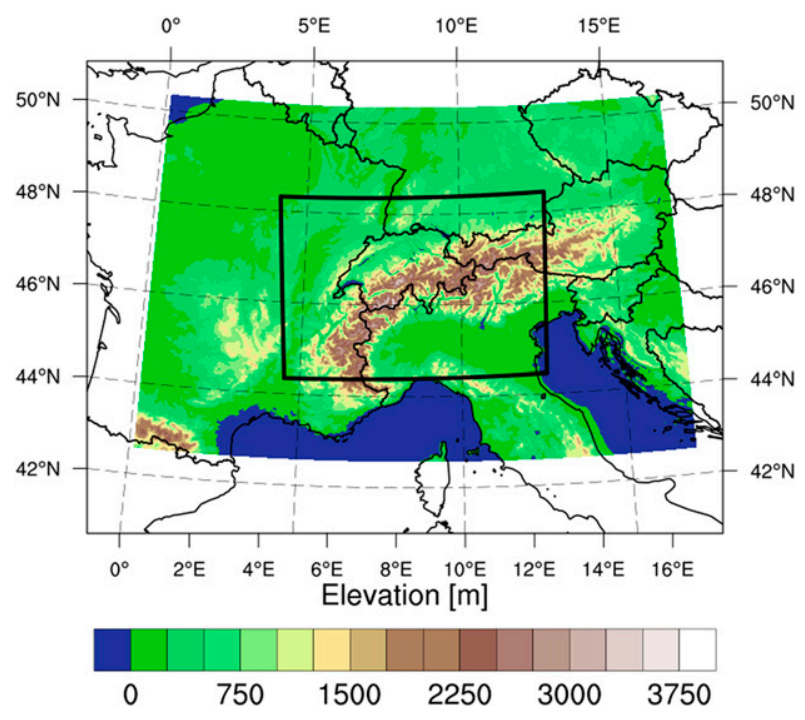

FIG. 1. COSMO-E domain (colored shading) and box used for the evaluation (black outline).

with $\chi$ the velocity potential, $\psi$ the streamfunction, and $\mathbf{k}$ the vertical unit vector. The irrotational wind $\mathbf{u}_{\text {irot }}$ corresponds to the first term in Eq. (1), and the nondivergent wind $\mathbf{u}_{\text {ndiv }}$ to the second term. According to this definition, it holds that

$$
\nabla \times \mathbf{u}_{\text {irot }}=0 \text { and } \nabla \cdot \mathbf{u}_{\text {ndiv }}=0 .
$$

Relating the divergence of the wind field to the Laplacian of the velocity potential

$$
\nabla \cdot \mathbf{u}=\nabla^{2} \chi
$$

allows us to compute $\chi$ by solving the Poisson equation ${ }^{1}$ with the boundary condition $\chi=0$, and hence to derive the zonal and meridional components of the irrotational wind:

$$
u_{\text {irot }}=\frac{\partial \chi}{\partial x} \quad \text { and } \quad v_{\text {irot }}=\frac{\partial \chi}{\partial y} .
$$

Likewise, the nondivergent wind can be derived from vorticity and the streamfunction. Since, however, solving the Poisson equation is computationally expensive, the nondivergent wind is derived simply as the residual of the full wind and the irrotational wind:

$$
u_{\text {ndiv }}=u-u_{\text {irot }} \text { and } \quad v_{\text {ndiv }}=v-v_{\text {irot }},
$$

which was found to be an appropriate approach by performing corresponding sensitivity tests (not shown).

\footnotetext{
${ }^{1}$ This is accomplished technically by the successive overrelaxation (SOR) scheme following Press et al. (1992).
} 

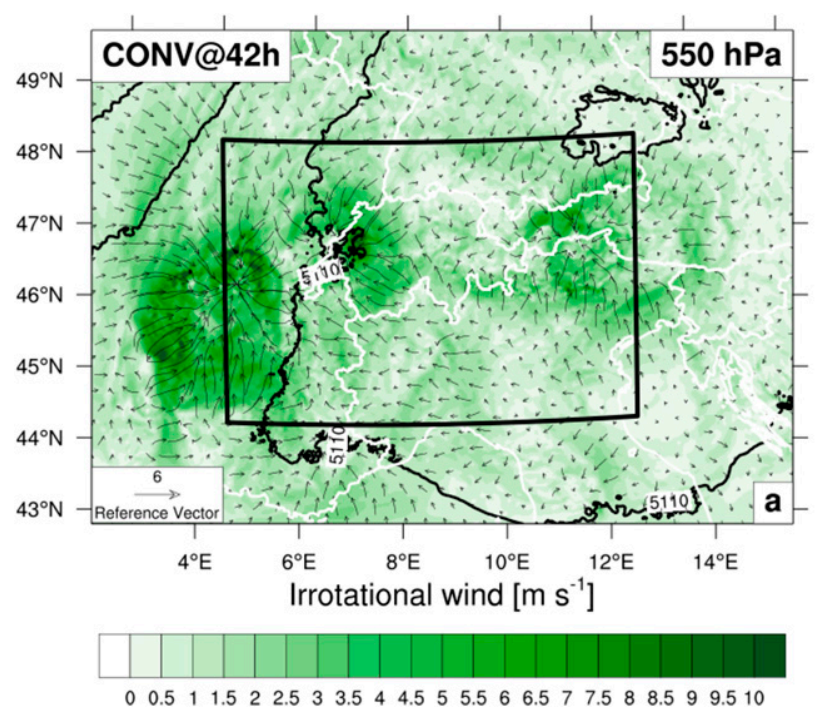

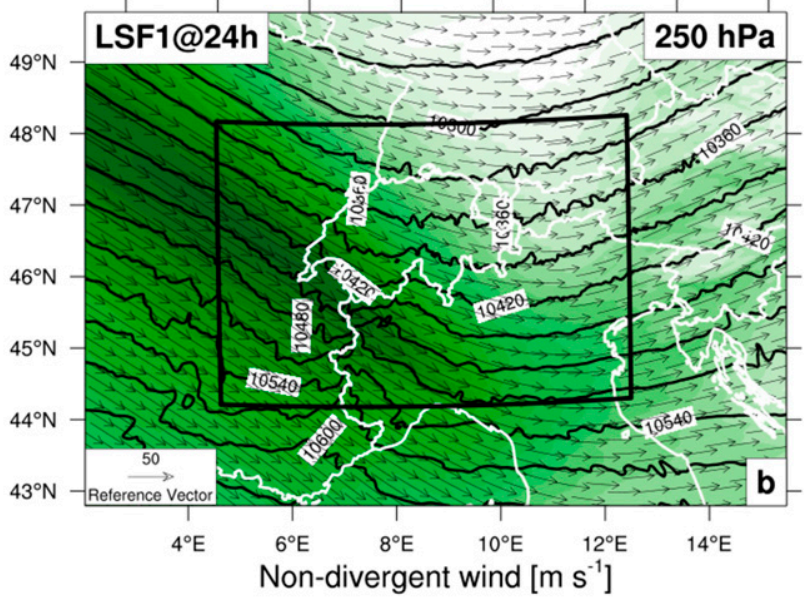

252729313335373941434547495153555759616365

FIG. 2. Maps of (a) irrotational wind speed (colors) and geopotential height (contours) at $550 \mathrm{hPa}$ for CONV at forecast hour 42 and (b) nondivergent wind speed and geopotential height at $250 \mathrm{hPa}$ for LSF1 at forecast hour 24 . Both plots show the control member. The black box is as in Fig. 1.

Note that in general both the nondivergent and irrotational winds can comprise a deformation flow component (Xu et al. 2011; Holton and Hakim 2013, 279-323). However, with our choice of the boundary conditions there is no harmonic component of $\chi$ and therefore the irrotational wind is purely divergent. Due to construction, the nondivergent wind then contains the rotational and deformation flow components.

Figure 2 illustrates both wind components for two case studies that are introduced in more detail in section $2 \mathrm{~d}$. The irrotational wind is shown for $550 \mathrm{hPa}$ at forecast hour 42 for the case CONV, which is characterized by convective summertime precipitation in Switzerland, while the nondivergent wind is depicted for $250 \mathrm{hPa}$ at forecast hour 24 for the case LSF1, where frontal precipitation caused flooding in Switzerland. As will be discussed later, the regions of increased irrotational wind speed (Fig. 2a) are associated with moist convection (e.g., over eastern France and western Austria). Nondivergent wind speeds (Fig. 2b) are particularly high in the region over eastern France, where the large horizontal pressure gradient indicates an intense upperlevel jet stream.

\section{c. Difference kinetic energy}

To study the growth of tiny perturbations in their simulations, Zhang et al. (2002) quantified differences between a perturbed and an unperturbed simulation by means of the DKE. In our study, DKE is defined as the sum of the squared differences between ensemble members and the ensemble mean of the horizontal wind components, calculated separately for the irrotational and nondivergent wind components; for instance,

$$
\begin{aligned}
\operatorname{DKE}_{\text {ndiv }}= & \frac{1}{2 M N} \sum_{j}^{M}\left\{\sum _ { i } ^ { N } \left[\left(u_{\text {ndiv }, i, j}-\bar{u}_{\text {ndiv }, j}\right)^{2}\right.\right. \\
& \left.\left.+\left(v_{\text {ndiv }, i, j}-\bar{v}_{\text {ndiv }, j}\right)^{2}\right]\right\},
\end{aligned}
$$

where $\bar{u}$ and $\bar{v}$ denote the ensemble-mean zonal and meridional wind, $i=1, \ldots, N$ the number of ensemble members, and $j=1, \ldots, M$ the total number of grid points over which DKE is averaged horizontally and vertically. The factor $1 / 2$ arises from the definition of kinetic energy. The value of $\mathrm{DKE}_{\text {irot }}$ is derived analogously.

DKE is evaluated in a box centered over Switzerland, which is roughly 4 times smaller than the full COSMO-E domain (Fig. 1). This does not only preclude detrimental impacts from the boundary zone but mostly serves to allow for comparison of DKE with the predictability level of precipitation, which is derived by means of the convective time scale $\tau$ for the region of Switzerland, as described in the following section.

\section{d. Case studies}

To study the time evolution of DKE, the three case studies CONV, LSF1, and LSF2 are selected with the purpose of covering different characteristics of precipitation events affecting Switzerland and described in some more detail in our companion paper (Klasa et al. 2018). 
Large-scale forcing (LSF) plays a dominant role in determining the weather situation over Switzerland for LSF1 and LSF2, while local processes are equally important for the convective case (CONV). During the 4-day forecasting period of the CONV case, warm and moist low-level air masses extend over central Europe (Fig. 3a), in the presence of an upper-level ridge (Fig. 3b) and weak vertical wind shear (Fig. 4a), favoring locally triggered, evening summertime convection on each of the 4 days. Low-frequency peak values embedded in surroundings of less intense precipitation (Fig. 5a) characterize the spotty field of the convective showers, illustrated in Fig. $6 \mathrm{~g}$ for the control member at forecast hour 18 (2000 local time), when showers are located over the Alps and in northeastern Switzerland.

A large, longitudinally extended gradient in equivalent potential temperature extends over Europe for the LSF1 case (Fig. 3c), accompanied by strong westerlies at upper levels (Fig. 3d) and therewith significant vertical wind shear (Fig. 4b). Precipitation in Switzerland is forced by a sequence of frontal passages, causing a more even distribution of the precipitation values (Fig. 5b). Higher precipitation amounts are located along the northern Swiss border and the northern Alpine slope, shown by the hourly precipitation field of the control member at forecast hour 12 (Fig. 6h).

During the case LSF2, the synoptic conditions over Switzerland are influenced by the large gradient in equivalent potential temperature (Fig. 3e) and the upperlevel trough over northwestern Europe (Fig. 3f). At the beginning of the forecasting period, the cold front approaches Switzerland from the northwest. With the arriving front, the vertical wind shear increases (Fig. 4c) and precipitation sets in, caused by prefrontal thunderstorm activity. Small precipitation amounts occur much more often than do the peak values (Fig. 5c), with precipitation occurring in the shape of small rainbands (Fig. 6i).

More details about the nature of the events and their predictability levels are obtained from two complementary measures: the convective time scale and the normalized precipitation spread. The convective time scale $\tau$ (Done et al. 2006; Keil et al. 2014) relates convective available potential energy (CAPE) to the precipitation rate (PREC),

$$
\tau \sim \frac{\mathrm{CAPE}}{\mathrm{PREC}},
$$

and therewith allows us to distinguish between equilibrium (small $\tau$ ) and nonequilibrium convection (large $\tau$ ), which are idealized concepts and the extremes of a continuous distribution (Zimmer et al. 2011). Large values of $\tau(>12 \mathrm{~h})$ indicate nonequilibrium convection (Keil et al. 2014), as CAPE can build up over a longer time scale and triggering mechanisms are required to overcome the energy barrier of CIN to release convection. In contrast, CAPE generated by the large-scale environment is readily consumed in the case of equilibrium convection and hence the values of $\tau$ are small $(<3 \mathrm{~h})$. Keil et al. (2014) qualitatively related the predictability level of precipitation to the value of $\tau$, which is categorized as "high" if $\tau<3 \mathrm{~h}$ and "low" if $\tau>12 \mathrm{~h}$.

For an ensemble of $N$ members with precipitation values $P_{i}(i=1, \ldots, N)$ and an ensemble mean precipitation $\bar{P}$, normalized precipitation spread $P_{s}$ is defined as

$$
P_{s}=\frac{\sqrt{\frac{1}{N} \sum_{i}\left(P_{i}-\bar{P}\right)^{2}}}{\bar{P}},
$$

relating the spread of the ensemble to the ensemblemean intensity of the precipitation event, which reduces the frequency of large values in the case of very high precipitation amounts.

Figure 6 shows these two qualitative measures of predictability for the three cases, that is, frequency distributions of hourly values of $\tau$ and of normalized precipitation spread as a function of forecast lead time. Convective-time-scale values are shown conditional on precipitation rates exceeding $1 \mathrm{~mm} \mathrm{~h}^{-1}$ and considering the ensemble members individually. For CONV, $\tau$ is large compared to both other cases, in particular at the times of precipitation onset (Fig. 6a). On day 3, $\tau$ reaches values of up to $20 \mathrm{~h}$, indicating a relatively low predictability level of precipitation. This is consistent with the comparatively frequent large values of normalized precipitation spread (in particular, on day 3; Fig. 6d). During the course of the daily precipitation events, the frequency maxima of $\tau$ are shifted from high values at the times of precipitation onset toward successively smaller values (Fig. 6a). In contrast, $\tau$ and $P_{s}$ are significantly smaller for LSF1 (Figs. 6b,e). Values of $\tau$ are consistently less than or equal to $0.5 \mathrm{~h}$ (Fig. $6 \mathrm{~b}$ ), and a significant fraction of the values is below $0.001 \mathrm{~h}$. Normalized precipitation spread values are below 3 for LSF1, a value that is frequently exceeded for CONV (Figs. 6d,e). According to both measures, the predictability level of precipitation for LSF2 lies in between that of both other cases (Figs. 6c,f). The peak in precipitation at hour 30 is associated with values of $\tau$ that are mostly smaller than $0.5 \mathrm{~h}$, while for the later peak at hour 39, $\tau$ ranges up to $6 \mathrm{~h}$. After the second day, no further precipitation is predicted by the ensemble. Note that Figs. 6a-e show the ensemble median precipitation to give a representative impression of the precipitation events. Time series thereof are shown explicitly for all ensemble members in Klasa et al. (2018). 

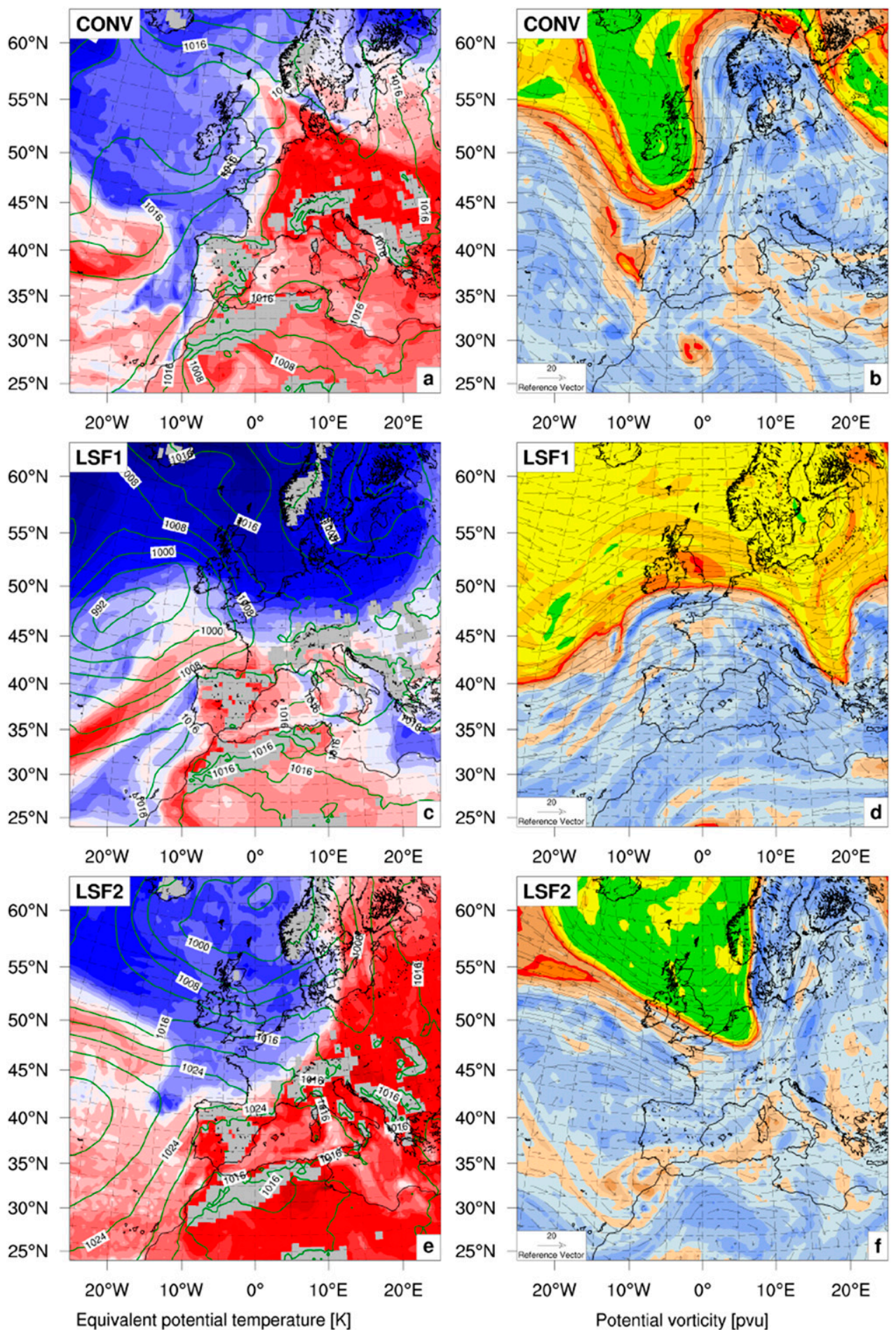

Equivalent potential temperature $[\mathrm{K}]$
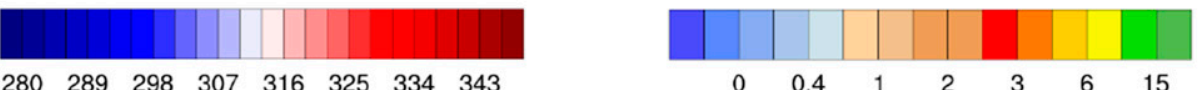

FIG. 3. Synoptic overview of (a),(b) CONV, (c),(d) LSF1, and (e),(f) LSF2 based on high-resolution analysis data from the ECMWF. (a),(c),(e) The 925-hPa equivalent potential temperature (K) (colored, with gray spots indicating orography higher than $925 \mathrm{hPa}$ ) and pressure at mean sea level (hPa; contours). (b),(d),(f) The potential vorticity (PVU, where $1 \mathrm{PVU}=10^{-6} \mathrm{~K} \mathrm{~m}^{2} \mathrm{~kg}^{-1} \mathrm{~s}^{-1}$; colored) and wind vectors, both at 325 K. Results are valid at (a),(b) 1200 UTC 10 Jun 2014, (c),(d) 1200 UTC 2 May 2015, and (e),(f) 1200 UTC 2 Jul 2016, which corresponds to forecast hour 36 for each case. 

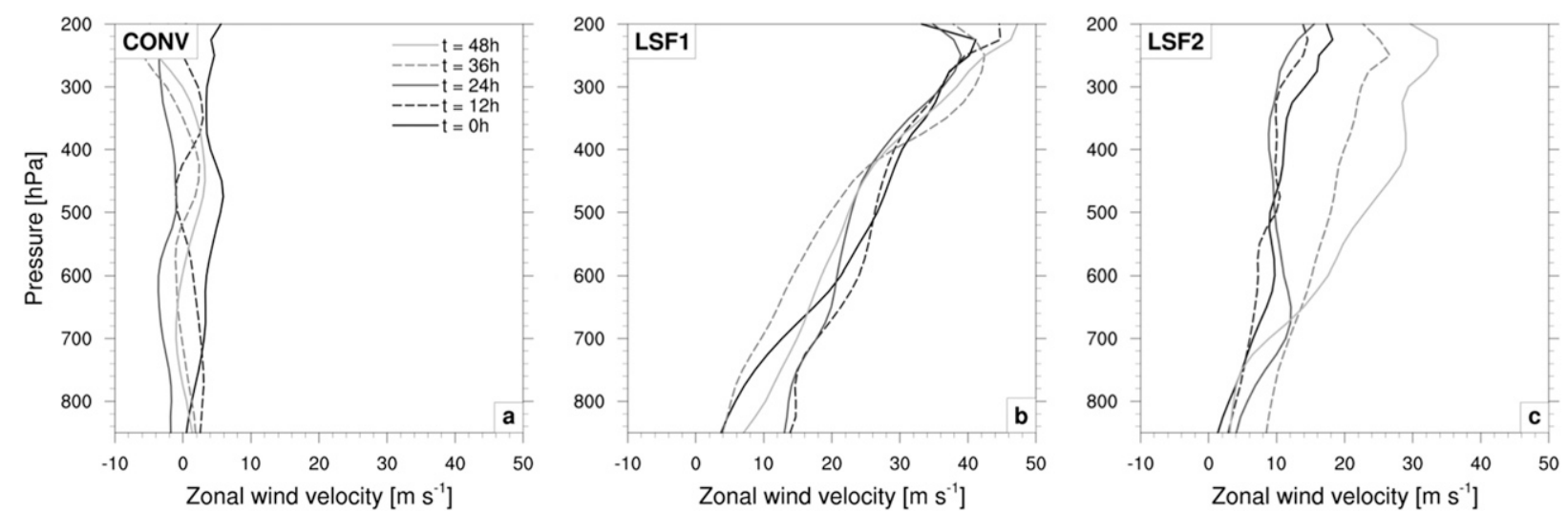

FIG. 4. Vertical profiles of the zonal wind velocity at five forecast lead times for the (a) CONV, (b) LSF1, and (c) LSF2 cases. The profiles are shown for the control member and averaged over the region of Switzerland.

In summary, the relative predictability level of precipitation is low for CONV, high for LSF1, and intermediate for LSF2, as consistently inferred with the aid of two independent qualitative measures of predictability.

\section{Ensemble variance of irrotational and nondivergent wind}

\section{a. The CONV case}

For CONV, Fig. 7a shows the time evolution of DKE for both wind components, averaged horizontally over the black box depicted in Fig. 1 and vertically between 850 and $200 \mathrm{hPa}$. For completeness, we mention that time series for difference total energy (i.e., including the temperature variance to the energy term) look very similar, indicating the kinetic energy terms dominate the temporal evolution of the difference total energy. Initially, $\mathrm{DKE}_{\text {ndiv }}$ is roughly a factor of 2 larger than

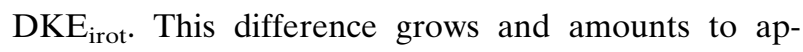
proximately an order of magnitude by the end of the time integration. During the first $16 \mathrm{~h}, \mathrm{DKE}$ of both wind components is augmented rapidly. The rapid growth of the initial errors was unanimously reported by previous predictability studies (e.g., Zhang et al. 2003, 2007; Selz and Craig 2015). After the initial increase, DKE $E_{\text {ndiv }}$ continues to grow, although at a slower rate, but $\mathrm{DKE}_{\text {irot }}$ remains at roughly the same level. However, a diurnal cycle is superimposed on the evolution of $\mathrm{DKE}_{\text {irot }}$, of which the peak times around 1800 UTC on each day correspond to the times of moist convective activity (Fig. 6a), preceded by high CAPE (Fig. 7g). The occurrence of moist convection constitutes the essential ingredient for rapid error growth (Zhang et al. 2003; Walser et al. 2004; Hohenegger et al. 2006; Hohenegger and Schär 2007; Zhang et al. 2007; Selz and Craig 2015). To confirm the presence of moist convection, Figs. $8 \mathrm{a}$ and $8 \mathrm{~g}$ show the 95th percentile, considering all ensemble members within the evaluation box, of vertical
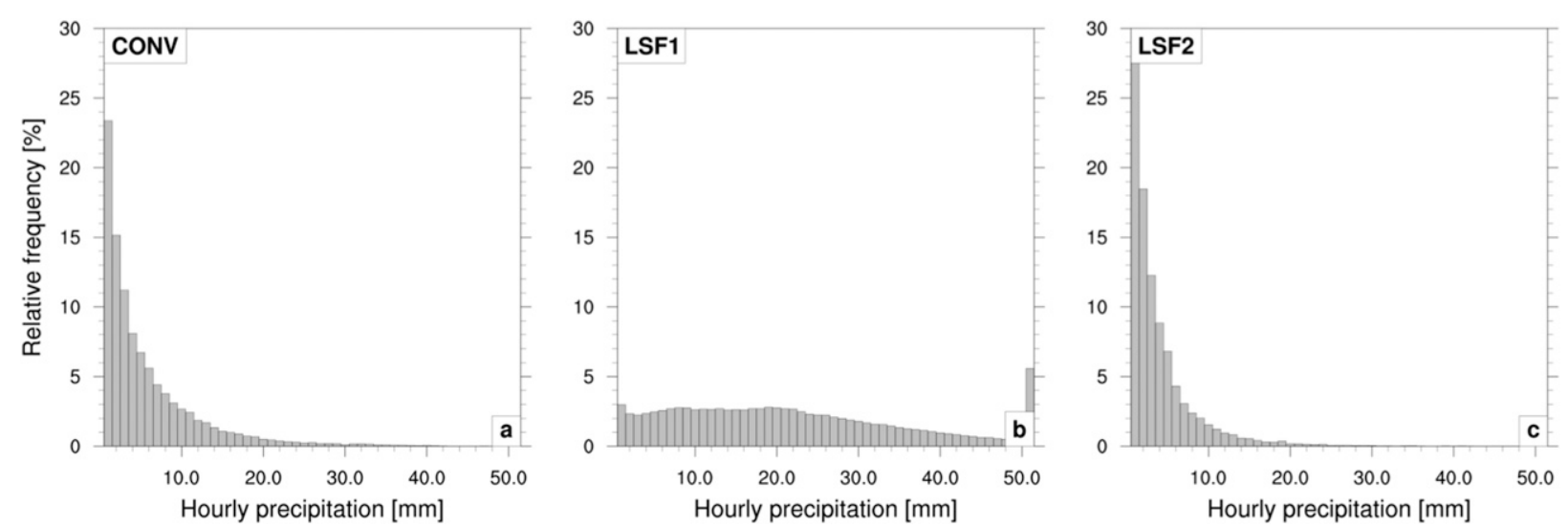

FIG. 5. Gridpoint-based frequency distributions of hourly precipitation rates, retrieved from hourly model output of the control member, considering the region of Switzerland and all forecast lead times, for the (a) CONV, (b) LSF1, and (c) LSF2 cases. 

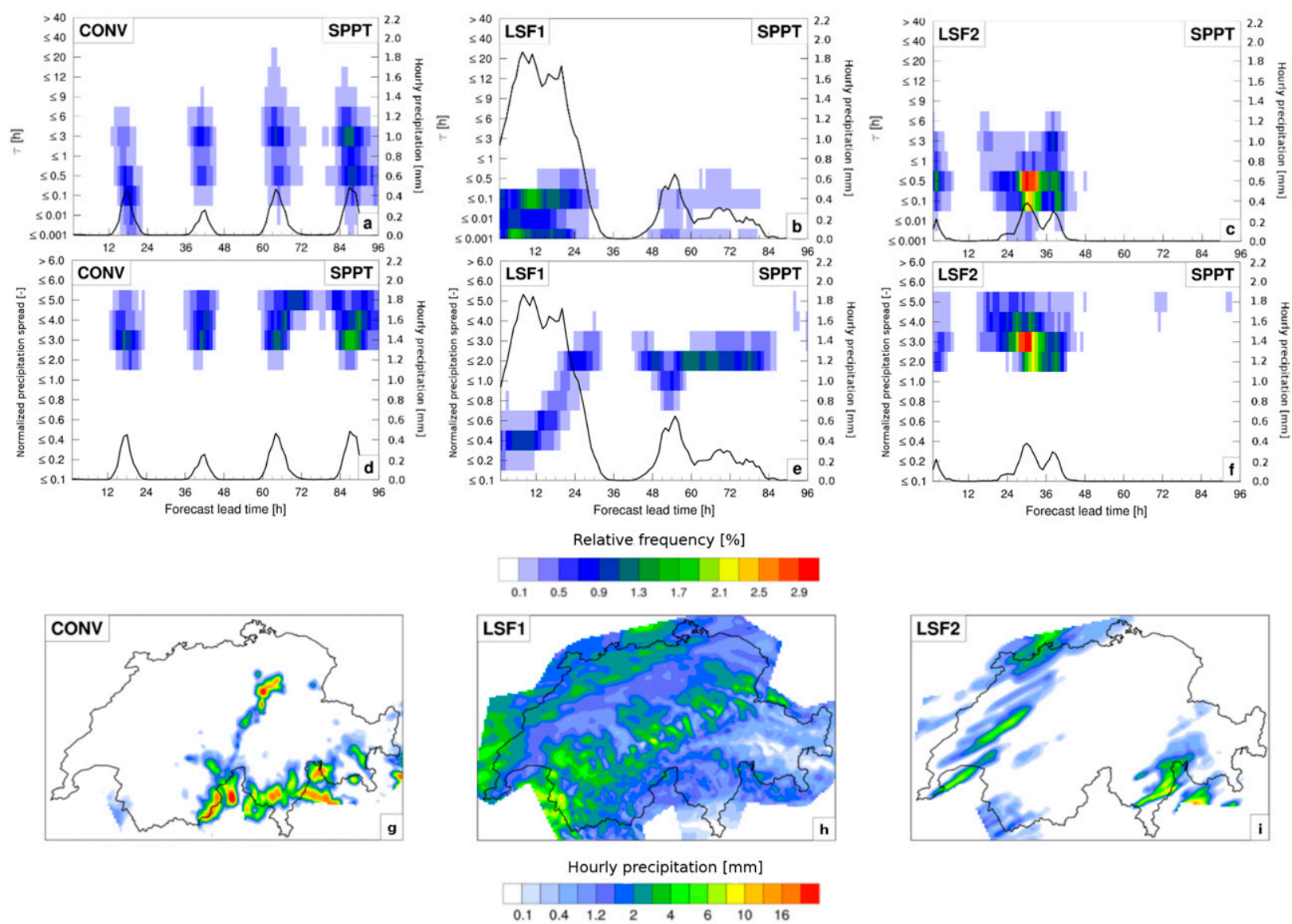

FIG. 6. (a)-(c) Time evolution of hourly frequency distributions of the convective time scale $\tau$ (colored) and of ensemble-median precipitation averaged over Switzerland (black line, right-hand-side $y$ axis). The plots are based on data from the experiments with SPPT and evaluated for the region of Switzerland. (d)-(f) Time evolution of frequency distributions of normalized spread of hourly precipitation (colored shading), including all grid points and time steps with an ensemble-mean precipitation rate exceeding $0.1 \mathrm{~mm} \mathrm{~h}{ }^{-1}$. The black line shows the ensemble-median precipitation averaged over Switzerland (right-hand-side $y$ axis) and data are taken from the SPPT experiment. (g)-(i) Maps of hourly precipitation from the control member, for CONV at forecast hour 18, LSF1 at hour 12, and LSF2 at hour 30, respectively.

wind and relative humidity, as a function of time and height. The white, vertical lines mark 1800 UTC on each day, prior to which strong vertical motions extend throughout the troposphere and co-occur with relatively moist air masses in the upper troposphere. In between two convective periods, the vertical wind is much smaller and the troposphere drier, in particular at the upper levels.

Figure 9 shows vertically resolved time series of $\mathrm{DKE}_{\text {irot }}$ (equivalent to Fig. 7a, except that there is no vertical averaging in Fig. 9). The signature of the daily variations extends throughout the troposphere, with maxima around 550 and $250 \mathrm{hPa}$, respectively. These two pressure levels embrace a layer of strong uppertropospheric updrafts (Fig. 8a), which go along with convergence below and divergence above due to continuity, eventually increasing the value of $\mathrm{DKE}_{\text {irot }}$. Regions of strong upper-level divergence (Figs. 10a,b) co-occur with regions of strong midtropospheric convergence over (south)eastern France, Switzerland, and western Austria (Figs. 10c,d). Upward motion at $400 \mathrm{hPa}$ is also enhanced in these regions, as are the values of $\mathrm{DKE}_{\text {irot }}$ at the upper- and midtropospheric levels (Fig. 10). (The magnitude of the 550-hPa irrotational wind at hour 42 is explicitly depicted in Fig. 2a, which corresponds to Fig. 10c.) The collocation of these features is a strong indication that the growth of the ensemble variance is related to updrafts and horizontal wind convergence and divergence, respectively. In addition, the affected regions are associated with deep convective clouds located over (south)eastern France, Switzerland, and western Austria, as shown by the satellite images in Fig. 11.

Consequently, the diurnal variation of $\mathrm{DKE}_{\text {irot }}$ (Figs. 7a and $9 \mathrm{a}$ ) is related to moist convective activity, corroborated 

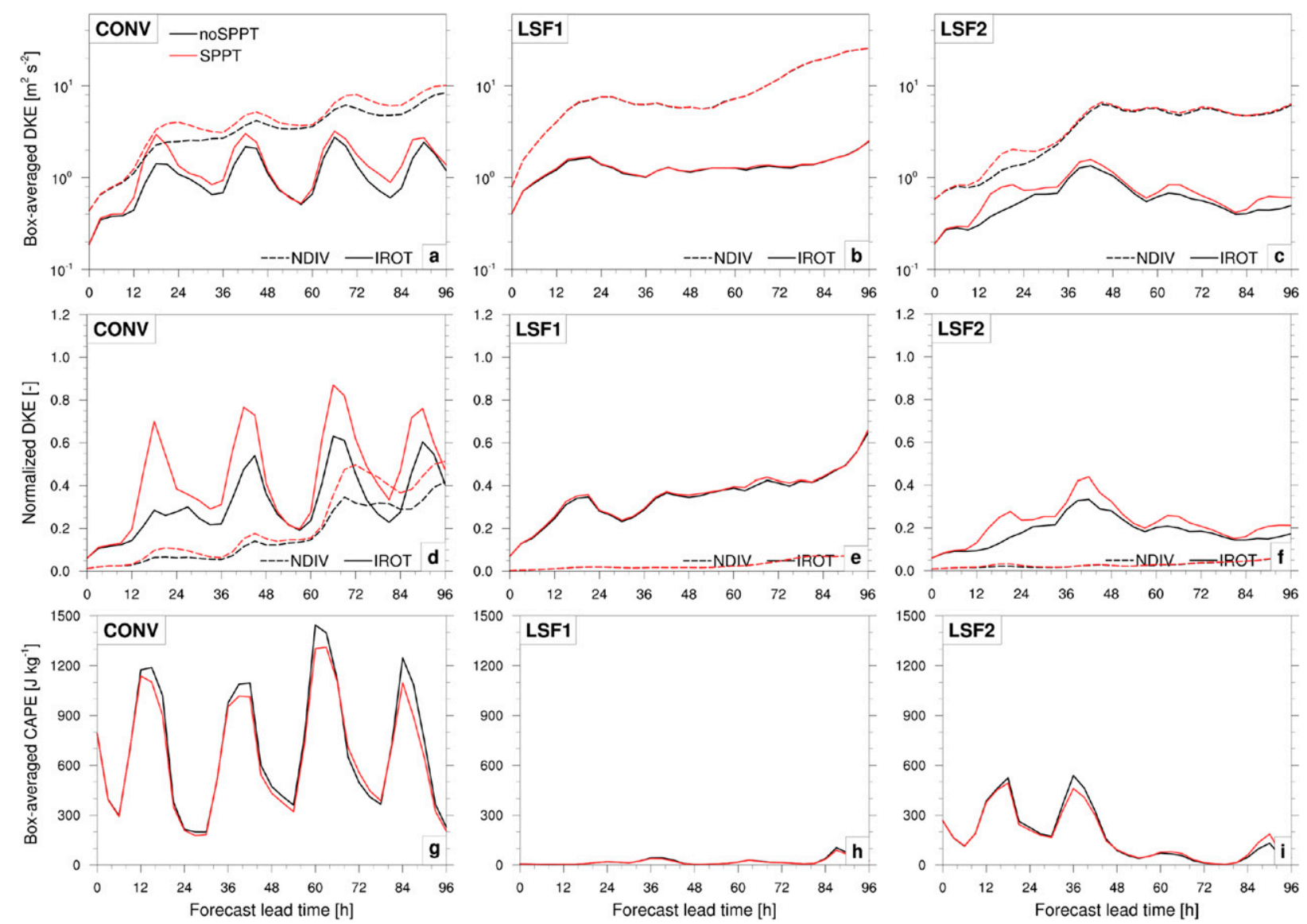

FIG. 7. Time series of (a)-(c) DKE and (d)-(f) DKE normalized by the ensemble-mean KE of the respective experiments, for the irrotational (solid) and nondivergent wind (dashed), and (g)-(i) of the ensemble-mean CAPE, for the (a),(d),(g) CONV, (b),(e),(h) LSF1, and (c),(f),(i) LSF2 cases. CAPE is averaged over the black box depicted in Fig. 1, as is DKE, which in addition is averaged vertically between 850 and $200 \mathrm{hPa}$.

by its spatiotemporal correlation to convergence and divergence (Fig. 10), updrafts (Fig. 8a), relative humidity (Fig. 8g), high CAPE (Fig. 7g), deep convective clouds (Fig. 11), and precipitation associated with a low predictability level (Fig. 6a).

A diurnal cycle is also superimposed on the steadily increasing $\mathrm{DKE}_{\text {ndiv }}$, with peak times several hours later than for DKE irot (Figs. 7a and 9d). The time shift of the diurnal cycle indicates that a part of $\mathrm{DKE}_{\text {irot }}$ is transferred to or triggers an increase in $\mathrm{DKE}_{\text {ndiv }}$, which is additionally suggested by spatial coherency. Figure 12 shows maps of upper-level $\mathrm{DKE}_{\text {irot }}$ and $\mathrm{DKE}_{\text {ndiv }}$ at consecutive times corresponding to one diurnal cycle. Initially (Fig. 12a), DKE irot $_{\text {has local maxima in the SW }}$ and NE of the box. Subsequently, it spreads out over a larger area (Figs. 12b,c), before it decreases successively (Figs. 12d-f). Values of DKE $E_{\text {ndiv }}$ increase in regions of enhanced $\mathrm{DKE}_{\text {irot }}$ (Fig. 12b), which affects increasingly larger regions. When $\mathrm{DKE}_{\text {irot }}$ vanishes, the spatial extent of enhanced $\mathrm{DKE}_{\text {ndiv }}$ decreases as well. The latter remains mostly restricted to regions of increased $\mathrm{DKE}_{\text {irot }}$, illustrated for instance by the decrease in DKE of both components south of Switzerland from Figs. 12d to 12e. A similar spatial coherency is assumed to be relevant also at lower levels, as suggested by Fig. 9d, which shows, at the end of the first day of the forecast, increased $\mathrm{DKE}_{\text {ndiv }}$ around $600 \mathrm{hPa}$, occurring also with the time shift of several hours compared to DKE irot.

As a short summary, $\mathrm{DKE}_{\text {irot }}$ exhibits a diurnal cycle driven by moist convective activity. $\mathrm{DKE}_{\text {ndiv }}$ steadily increase, with a superimposed diurnal cycle shifted by a few hours compared to $\mathrm{DKE}_{\text {irot }}$. The spatiotemporal correlation of DKE of both wind components suggests that $\mathrm{DKE}_{\text {irot }}$ induces the daily variations of $\mathrm{DKE}_{\text {ndiv }}$.

Normalizing $\mathrm{DKE}_{\text {irot }}$ and $\mathrm{DKE}_{\text {ndiv }}$ by the respective ensemble-mean kinetic energy values $\left(\mathrm{KE}_{\text {irot }}\right.$ and $\mathrm{KE}_{\text {ndiv }}$, respectively) allows us to estimate the ensemble variability independently of the magnitude of the background flow (Fig. 7d). Normalized DKE $E_{\text {ndiv }}$ is smaller than normalized $\mathrm{DKE}_{\text {irot}}$, in particular during the first 2 days of 

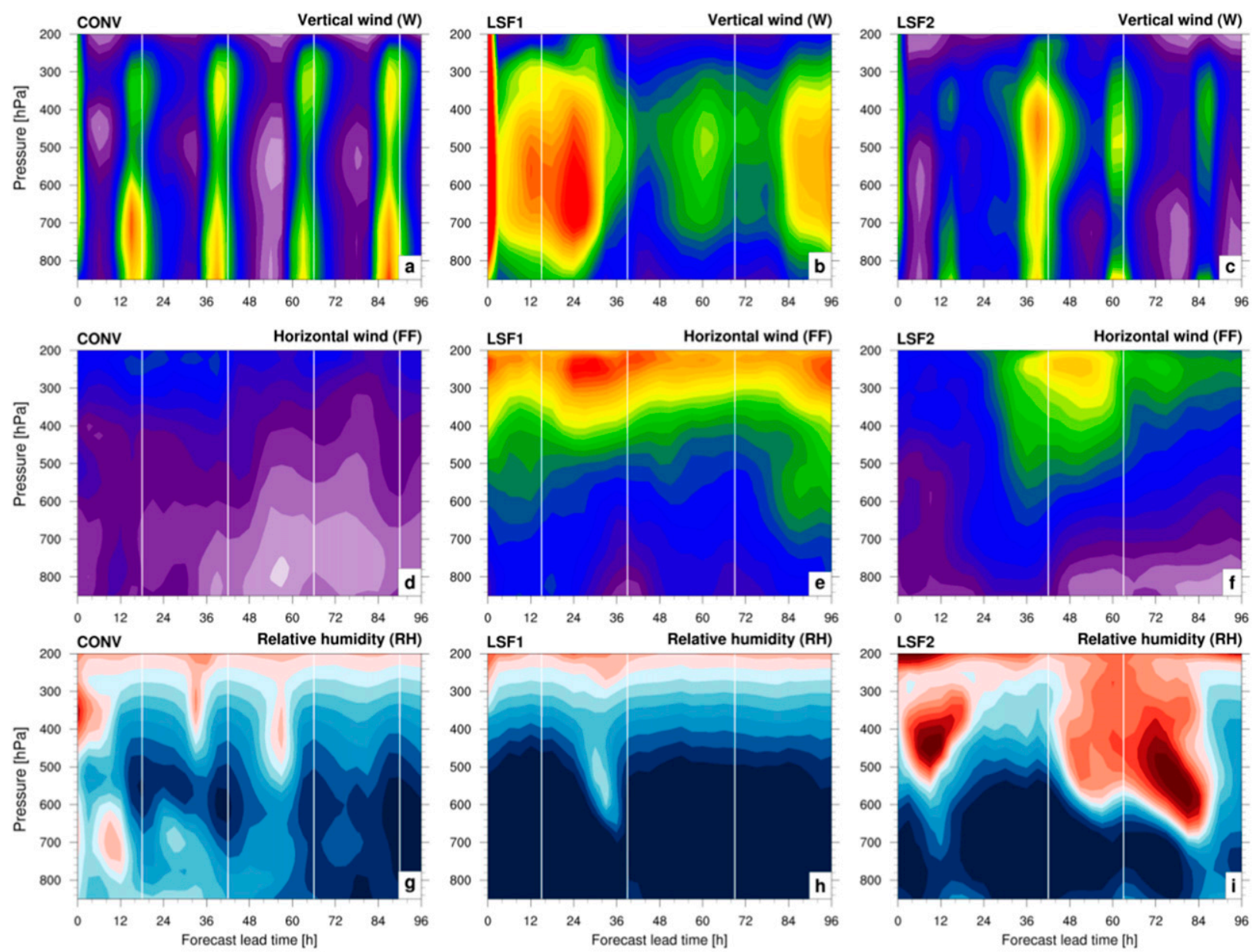

Horiz. W 95th $\left[10^{-1} \mathrm{~m} \mathrm{~s}^{-1}\right]$

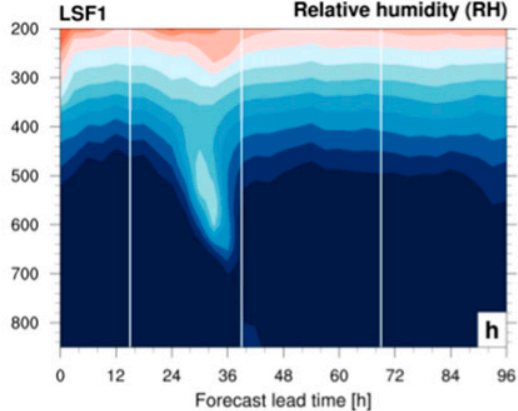

Horiz. FF 95th $\left[\mathrm{m} \mathrm{s}^{-1}\right]$

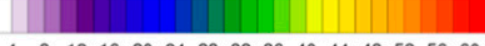

$\begin{array}{lllllllllllllll}4 & 8 & 12 & 16 & 20 & 24 & 28 & 32 & 36 & 40 & 44 & 48 & 52 & 56 & 60\end{array}$

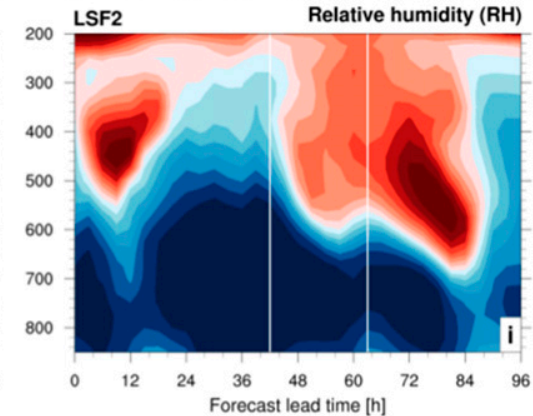

Horiz. RH 95th [\%]

253035404550556065707580859095

$\begin{array}{lllllllllllllll}0.5 & 1 & 1.5 & 2 & 2.5 & 3 & 3.5 & 4 & 4.5 & 5 & 5.5 & 6 & 6.5 & 7 & 7.5\end{array}$

FIG. 8. Pressure-time contour plot of the horizontal 95th percentile, considering all ensemble members within the evaluation box, of (a)-(c) vertical and (d)-(f) horizontal winds, and (g)-(i) relative humidity for the experiments with SPPT for the (a),(d),(g) CONV, (b),(e),(h) LSF1, and (c),(f),(i) LSF2 cases.

the integration. Thus, absolute $\mathrm{DKE}_{\text {ndiv }}$ is large because it is closely related to a strong nondivergent background flow. Normalized $\mathrm{DKE}_{\text {irot }}$ exhibits a diurnal cycle, which is also evident for normalized $\mathrm{DKE}_{\text {ndiv }}$, including the above-discussed time shift. Normalized $\mathrm{DKE}_{\text {ndiv }}$ increases stepwise on a daily basis. In contrast to normalized $\mathrm{DKE}_{\text {irot }}$, normalized $\mathrm{DKE}_{\text {ndiv }}$ decreases only slightly after the daily increase. Figure $7 \mathrm{~d}$ strongly indicates that $\mathrm{DKE}_{\text {irot }}$ provides a significant contribution to the increase in $\mathrm{DKE}_{\text {ndiv }}$, which is independent of the background flow. Idealized studies by Weyn and Durran (2017) showed a significant impact of mesoscale convective systems on $\mathrm{DKE}_{\text {ndiv }}$, which is in line with our findings.

Contrasting $\mathrm{DKE}_{\text {irot }}$ for noSPPT and SPPT shows significant differences during the peak times of convective activity (Fig. 7a), when the SPPT ensemble exhibits a larger variability than the noSPPT ensemble, while in between the convective periods, the differences become relatively small, in particular after the second day when $\mathrm{DKE}_{\text {irot }}$ values for noSPPT and SPPT are equal. This applies similarly to $\mathrm{DKE}_{\text {ndiv }}$, which is larger for SPPT during the peak times that occur somewhat later than for DKE $_{\text {irot. }}$ The differences among the experiments apply similarly to normalized DKE (Fig. 7d).

In summary, for the convective case, ensemble variance of the horizontal wind exhibits a diurnal cycle, which is forced by solar energy as corroborated by the close relation of wind variance and CAPE. While the variance of the irrotational wind fluctuates around a mean level, the diurnal cycle in the variance of the nondivergent wind is superimposed on an increasing trend, with strong indications that a part of the 

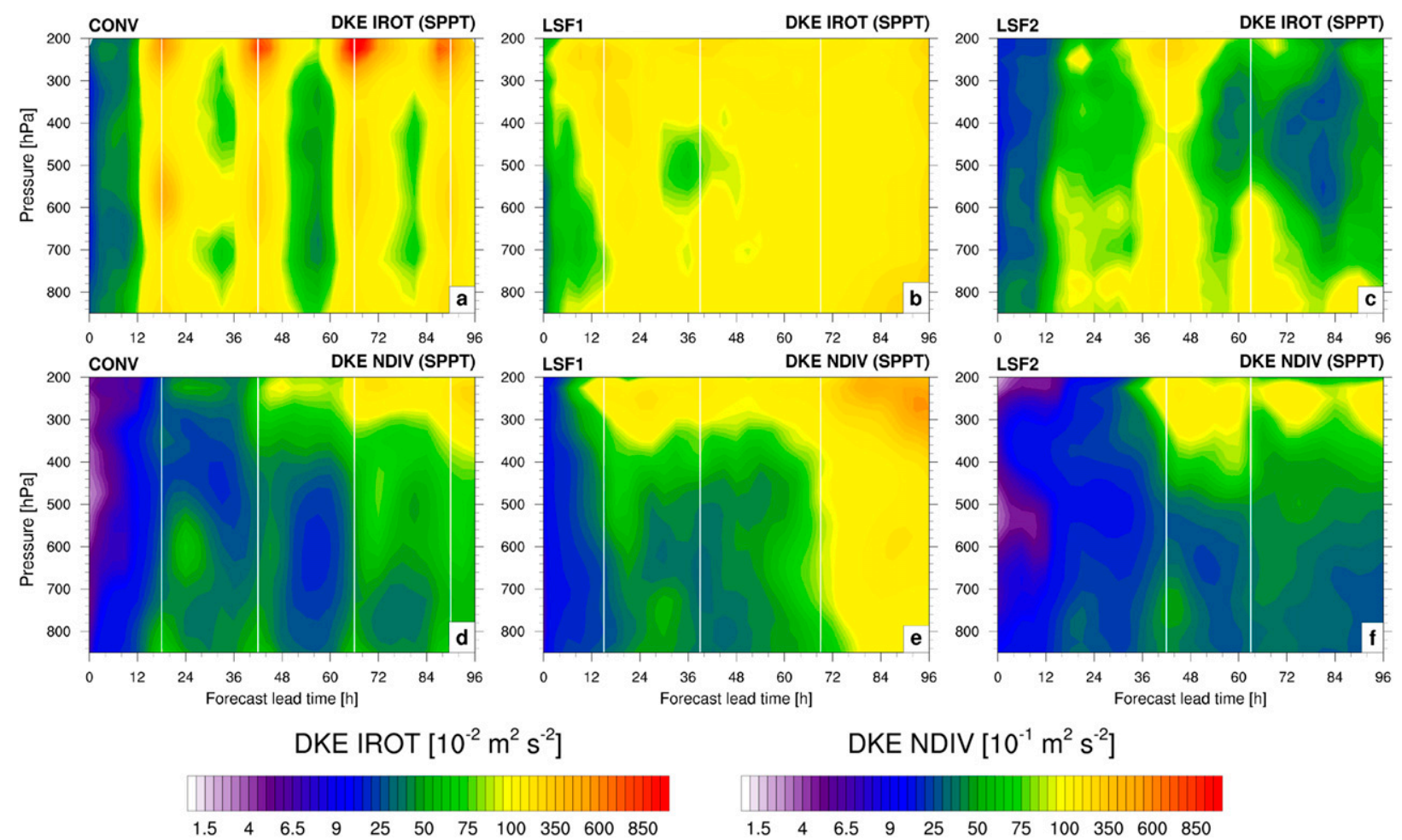

FIG. 9. Pressure-time contour plot of box-averaged DKE of the (a)-(c) irrotational and (d)-(f) nondivergent winds. The plots are shown for the experiments with SPPT for the (a),(d) CONV, (b),(e) LSF1, and (c),(f) LSF2 cases. The white vertical lines indicate particular forecast hours and are referred to in the text. Note that the units for the color bars of the irrotational and nondivergent winds differ by one order of magnitude.

variability is being transferred from the irrotational to the nondivergent wind. Consequently, for both wind components, ensemble variability induced by moist convection does not increase continuously during the forecast integration but decreases on a daily time scale. Similar analyses for the LSF1 and LSF2 events will further illustrate the role of moist convection and the synoptic-scale control in governing the growth dynamics of ensemble variance.

\section{b. The LSF1 case}

The LSF1 case is, in contrast to CONV, characterized by strong large-scale advection and a significantly higher predictability level of precipitation (Fig. 6b), coinciding with very low CAPE (Fig. 7h). As for CONV, DKE ndiv $_{\text {is }}$ initially a factor of 2 larger than $\mathrm{DKE}_{\text {irot }}$, while it is roughly an order of magnitude larger by the end of the forecast integration (Fig. 7b). The overall evolution of DKE for LSF1 is similar to that for CONV. However, several aspects differ, among them are the absence of any variations on a diurnal time scale and the strong similarity of noSPPT and SPPT for DKE of both wind components. Figure $9 \mathrm{e}$ shows that large values of $\mathrm{DKE}_{\text {ndiv }}$ occur mostly in the upper troposphere, in particular during the first 2 days of the forecast, coinciding with high horizontal wind speeds (Fig. 8e). The relation of the nondivergent wind to the upper-level jet stream is illustrated in Fig. 2b. Normalized DKE $E_{\text {ndiv }}$ remains low except for a small increase after $72 \mathrm{~h}$, while normalized $\mathrm{DKE}_{\text {irot }}$ steadily increases without any diurnal variations (Fig. 7e), suggesting a significant background-flow independent variability of the irrotational wind.

While there is a clear relation between vertical motions and $\mathrm{DKE}_{\text {irot }}$ for CONV, the strong updrafts and high values of relative humidity, for instance on the first day of LSF1 (Figs. 8b,h), do not induce any variation in DKE $_{\text {irot }}$ (Fig. 9b), which is due to the different mechanisms of forcing vertical motion. In contrast to CONV, for which forcing is due to local low-level convergence and thermodynamic instability, frontal upglide forced by the large-scale flow induces significant updrafts for LSF1. Since the latter is not associated with domaininternal convergence, the forcing is not reflected in the

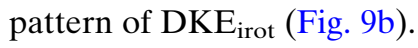

Consequently, the temporal increase in $\mathrm{DKE}_{\text {irot }}$ for LSF1 is not generated by moist convection as for CONV but is strongly determined by the large-scale flow. 

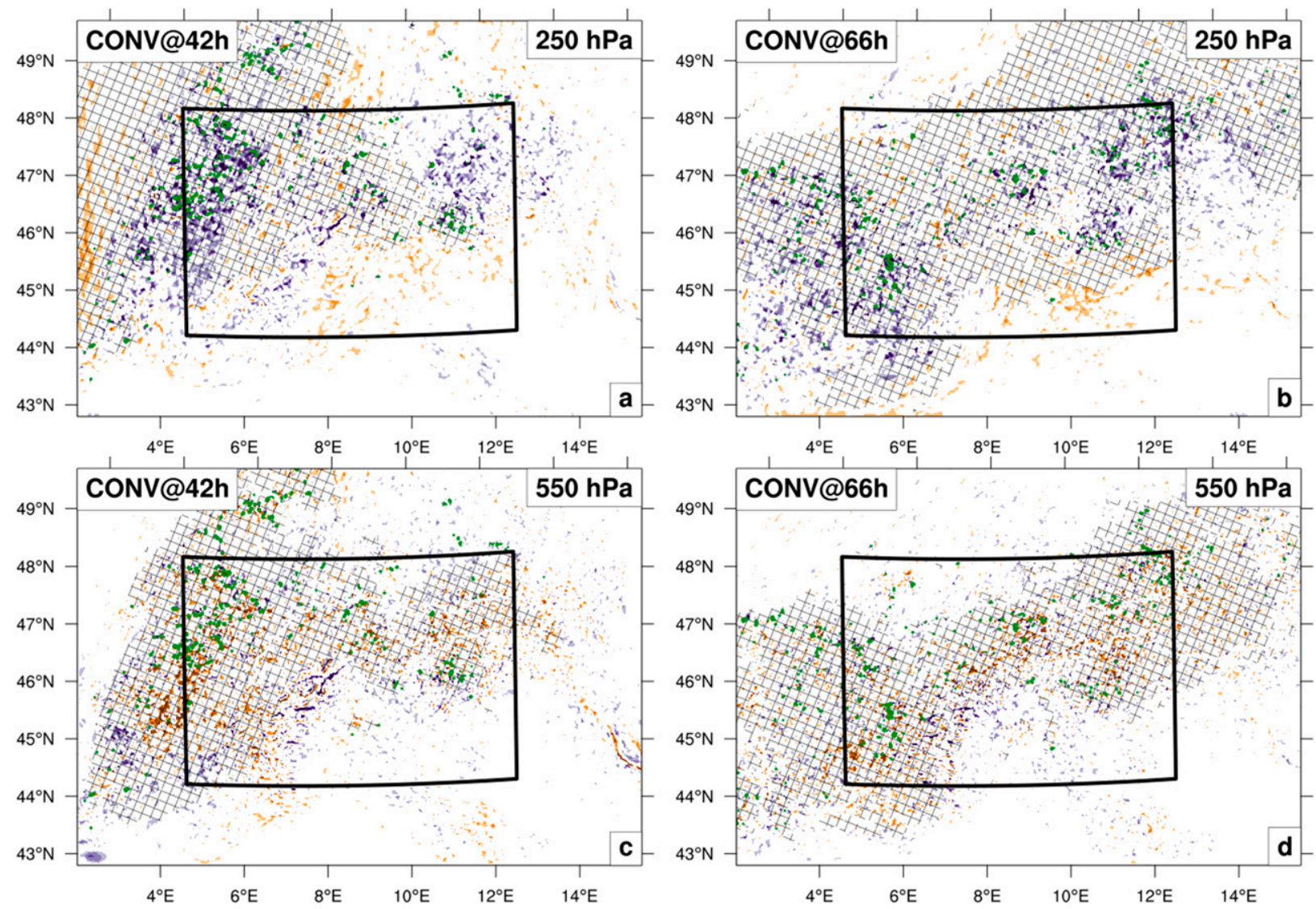

Divergence $\left[10^{-4} \mathrm{~s}^{-1}\right]$

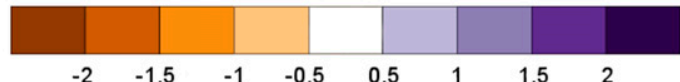

FIG. 10. Map of ensemble-mean divergence (colored shading) at (a),(b) 250 and (c),(d) $550 \mathrm{hPa}$ for DKE $\mathrm{E}_{\text {irot }}$ at $250 \mathrm{hPa}$ exceeding $6 \mathrm{~m}^{2} \mathrm{~s}^{-2}$ (hatching) and at $550 \mathrm{hPa}$ exceeding $1 \mathrm{~m}^{2} \mathrm{~s}^{-2}$ (hatching), respectively. The 400 -hPa ensemble-mean vertical wind of $0.8 \mathrm{~m} \mathrm{~s} \mathrm{~s}^{-1}$ is shown by green contours. The plots are shown for the experiment with SPPT for the CONV cases at forecast hours (a),(c) 42 and (b),(d) 66 .

\section{c. The LSF2 case}

For LSF2, initial values of box-averaged $\mathrm{DKE}_{\text {irot }}$ and $\mathrm{DKE}_{\text {ndiv }}$ differ by a factor of 3 (Fig. $7 \mathrm{c}$ ), while the difference amounts to roughly an order of magnitude by the end of the integration. The overall evolution of the DKE time series differs from those for CONV and LSF1. The steeper initial increase in DKE of both wind components extends over a significantly longer time period compared to both other cases. Afterward, $\mathrm{DKE}_{\text {ndiv }}$ remains constant and does not increase further, while $\mathrm{DKE}_{\text {irot }}$ decreases continuously. A diurnal cycle is superimposed on this overall trend of $\mathrm{DKE}_{\text {irot}}$, which is weaker than for CONV and related to increased CAPE (Fig. 7i). Some similar variations are also apparent for $\mathrm{DKE}_{\text {ndiv }}$, mostly with the time shift already reported for CONV. Differences among the experiments with and without SPPT are similar to LSF1 for DKE ${ }_{\text {ndiv }}$, except for larger values in SPPT on the first day. For $\mathrm{DKE}_{\text {irot, }}$, the differences are rather similar to CONV, with larger differences between noSPPT and SPPT at the peak times of the diurnal cycle.

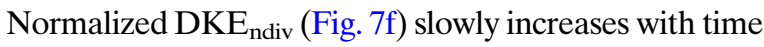
in a manner similar to that for LSF1 and is significantly smaller than normalized $\mathrm{DKE}_{\text {irot. }}$. The time evolution of the latter is qualitatively very similar to $\mathrm{DKE}_{\text {irot }}$ (Fig. 7c). Consequently, the variability in the nondivergent wind is mainly due to high wind velocities.

The diurnal variations superimposed on the general trend of $\mathrm{DKE}_{\text {irot }}$ are temporally correlated to updrafts (Fig. 8c), whose structure and strength differ from those in CONV. On day 2, the temporally extended updraft is related to the precipitation event (Fig. 6c), as shown by the high values of relative humidity in the lower troposphere in Fig. 8i. Thereafter, deep moist convective 

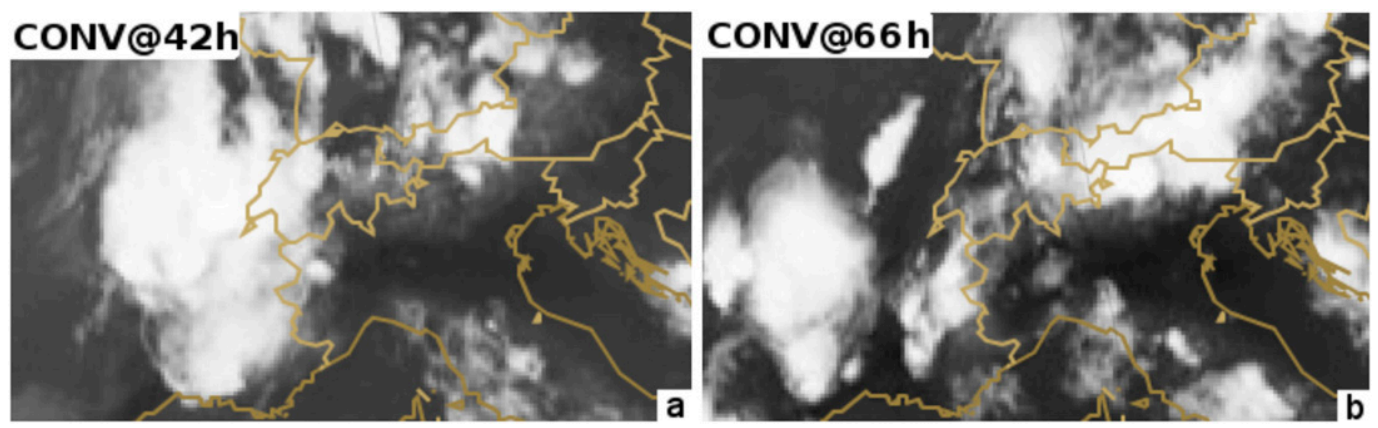

FIG. 11. Meteosat images from the EUMeTrain training project sponsored by EUMETSAT and valid at (a) 1800 UTC 10 Jun 2014 (CONV at forecast hour 42) and (b) at 1800 UTC 11 Jun 2014 (hour 66), cropped to fit the COSMO-E domain.

activity is damped by the postfrontal dry-air intrusion indicated by very low values of relative humidity.

In summary, compared to both other cases, LSF2 is a peculiar case due to the less steep initial increase in DKE of both wind components, and the subsequent stagnation of $\mathrm{DKE}_{\text {ndiv }}$ and decrease in $\mathrm{DKE}_{\text {irot }}$, though the latter still exhibits a weak diurnal cycle. The decrease in $\mathrm{DKE}_{\text {irot }}$ is associated with weak moist convective activity, as indicated by overall weaker updrafts compared to CONV and descending dry air masses. Moist convection is essential to maintaining DKE at all scales (Zhang et al. 2007), implying a decrease in DKE when moist convection vanishes, to which dissipation possibly contributes as well, though it may be sustained or may increase further in cases where it is advected from the domain boundaries (e.g., LSF1).

\section{Summary and conclusions}

For three cases with precipitation in Switzerland, we study the time evolution of the ensemble variance of the horizontal wind by investigating 4-day forecasts of the convection-permitting ensemble COSMO-E, which has been operational at MeteoSwiss since May 2016.
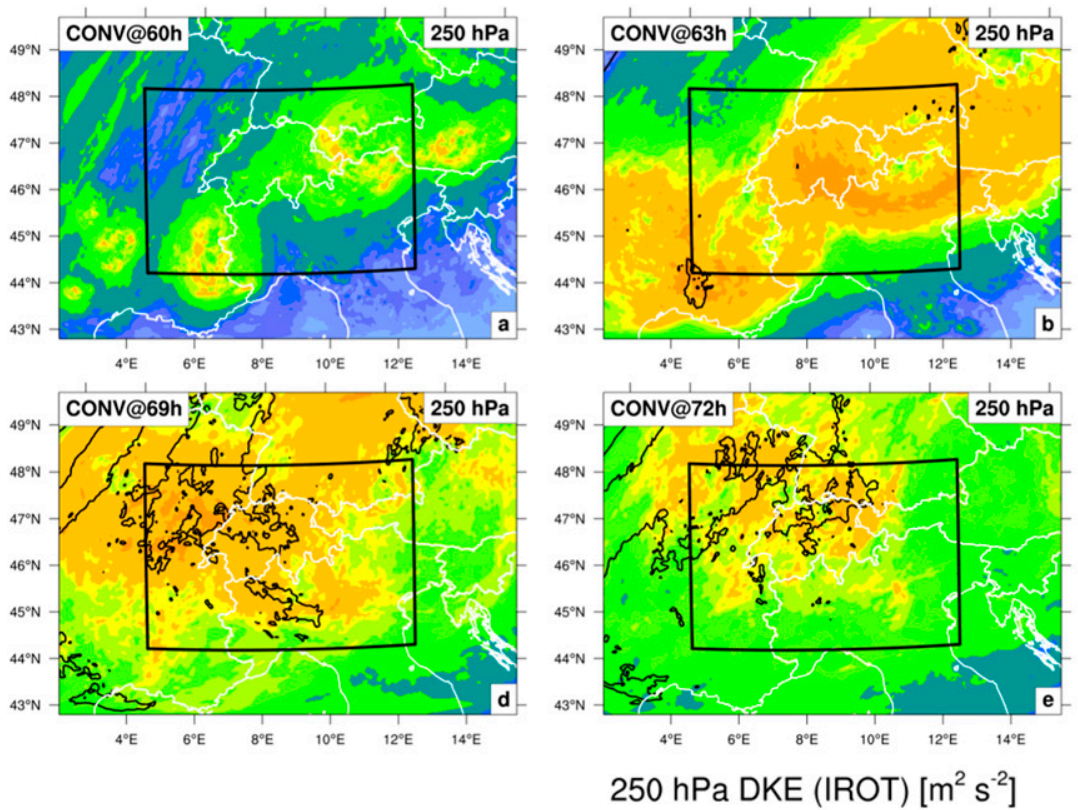
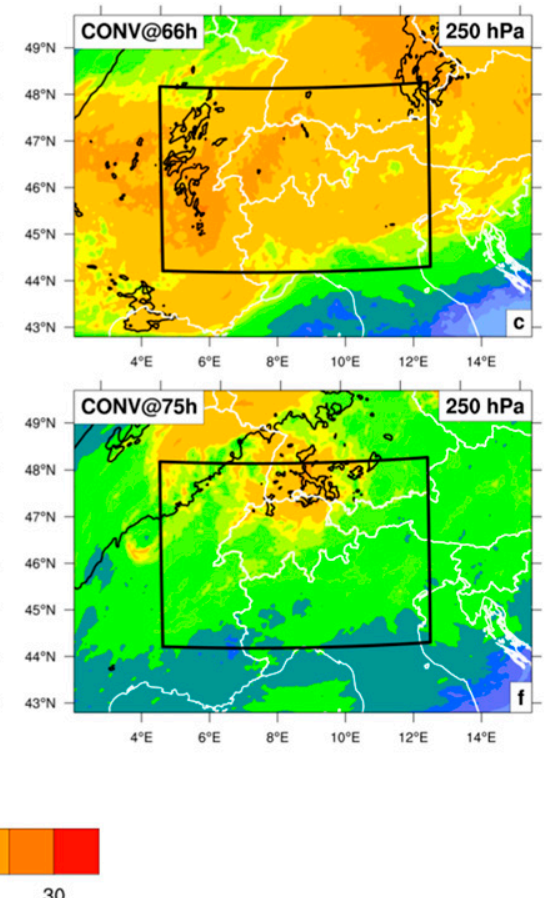

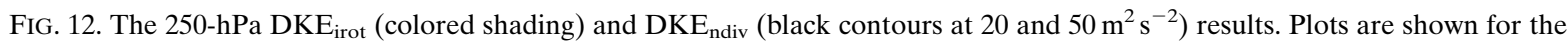
experiment with SPPT for the CONV case in 3-h increments from forecast hours (a) 60 to (f) 75. 
Ensemble variance, quantified by means of difference kinetic energy (DKE), is calculated for the irrotational $\left(\mathrm{DKE}_{\text {irot }}\right)$ and nondivergent wind $\left(\mathrm{DKE}_{\text {ndiv }}\right)$, which is motivated by their contrasting dynamical implications. While the irrotational wind is relevant most of all in convective systems where local convergence is crucial to generate strong updrafts inducing divergence aloft, the nondivergent wind can be associated primarily with the balanced flow, which is important at larger scales. Difference kinetic or total energy has been widely used in predictability studies to quantify error growth, for instance, by Zhang et al. (2002, 2003, 2007), Selz and Craig (2015), Nielsen and Schumacher (2016), and Weyn and Durran (2017).

The time evolution patterns of $\mathrm{DKE}_{\text {irot }}$ and $\mathrm{DKE}_{\text {ndiv }}$ are investigated for the three cases of contrasting predictability levels of precipitation. CONV is characterized by locally triggered convective precipitation on four consecutive days, exhibiting a relatively low level of predictability of precipitation. In contrast, the predictability level of precipitation is higher for LSF1, for which a strong large-scale control forces upward motion and heavy precipitation in Switzerland. LSF2 is a case of intermediate predictability of precipitation compared to both other cases and characterized by a weaker largescale advection than in LSF1, with precipitation in Switzerland related to prefrontal thunderstorm activity. The predictability levels of precipitation are closely related to the dynamics of the variance growth of the horizontal wind.

For CONV, DKE $E_{\text {irot }}$ exhibits a clear solar-forced diurnal cycle. During the daytime, moist convective activity leads to an increase in $\mathrm{DKE}_{\text {irot, }}$, which is temporally correlated to high-CAPE and high-precipitation periods with a low predictability level. However, ensemble variance does not continue to increase but decreases again when moist convective activity vanishes, in agreement with, for example, Zhang et al. (2006, 2007), implying a large-scale control on the growth of ensemble variance, as found by Nielsen and Schumacher (2016). The diurnal cycle also occurs for $\mathrm{DKE}_{\text {ndiv }}$ for which the daily peaks occur several hours later than for $\mathrm{DKE}_{\text {irot }}$. The diurnal, stepwise increase in the background-flow independent $\mathrm{DKE}_{\text {ndiv }}$ (i.e., $\mathrm{DKE}_{\text {ndiv }}$ normalized by the ensemble mean kinetic energy of the nondivergent flow) indicates that increased variability in the nondivergent wind is induced by increased variability in the irrotational wind, which might be in agreement with the geostrophic adjustment process highlighted by several previous studies (e.g., Schubert et al. 1980; Bierdel et al. 2017).

Moist convection is of key importance not only for increasing $\mathrm{DKE}_{\text {irot }}$, as illustrated by the CONV case, but also for maintaining its magnitude. The absence of moist convection in the second half of LSF2 leads to a continuous decrease in $\mathrm{DKE}_{\text {irot }}$, while $\mathrm{DKE}_{\text {ndiv }}$ remains constant during this period, which is dominated by the postfrontal dry-air intrusion restricting convective activity to lower-tropospheric altitudes.

The predictability level of precipitation is high for LSF1, which is due to the large-scale forcing of precipitation associated with low CAPE. Strong updrafts are not accompanied by a strong increase in $\mathrm{DKE}_{\text {irot }}$, since they are due to large-scale frontal ascent and not to a highly divergent and thermodynamically unstable flow, as for CONV. For LSF1, the large-scale control is crucial to fostering the growth of ensemble variance of both wind components.

Contrasting two experiments, noSPPT and SPPT, of which the latter applies the stochastically perturbed parameterization tendencies scheme (Buizza et al. 1999), shows that at the peak times of the diurnal cycle of convection, $\mathrm{DKE}_{\text {irot }}$ is larger for SPPT than for noSPPT, which applies similarly to $\mathrm{DKE}_{\text {ndiv }}$. During nonconvective episodes, DKE values of both wind components are mostly alike for the experiments.

From our study, we draw the following conclusions in response to the questions posed in the introduction:

- The overall time evolution of ensemble variance is constrained by the large scale either in the context of moist convection forced by solar energy or the synoptic-scale control imposed at the lateral domain boundaries.

- Under moist convective conditions, ensemble variance of the irrotational wind is augmented by convective activity and ceases when convection vanishes. This signal is to a lesser extent also present in the ensemble variance of the nondivergent wind, pointing to interactions between both wind components. At the peak times of moist convective activity, ensemble variance is clearly sensitive to uncertainties in the model physics, yet this does not alter the overall time evolution of ensemble variance.

- In the case of a strong synoptic-scale forcing for ascent, associated with a high predictability level of precipitation, uncertainties related to domain-internal physical processes hardly matter for the time evolution of ensemble variance of both wind components, which is determined by the synoptic-scale control. Note that a postfrontal dry-air intrusion also belongs to this category, as it is a synoptic-scale phenomenon that, in this case, impedes convection and decreases ensemble variance.

Consequently, the overall growth of ensemble variance is constrained by the large-scale flow, implying that the time horizon of convection-permitting ensemble 
forecasts is not limited to the first occurrence of moist convection, since the latter does not lead to incessantly diverging forecast integrations of the ensemble members. Therefore, a forecast range of several days is possible for limited-area models, which is in line with the study of Nielsen and Schumacher (2016). However, our conclusions rely upon a small number of case studies and do not cover the full range of meteorological conditions associated with precipitation in Switzerland, such as, for example, a case where the large-scale forcing constantly triggers convective activity, resulting in continuous spread growth (Nielsen and Schumacher 2016). Our study focused on the multiday range of ensemble forecasts and did not address the steep initial increase in ensemble variance of both wind components, which was investigated by a number of previous studies showing strongly limited predictability on small spatiotemporal scales (e.g., Zhang et al. 2003; Walser et al. 2004; Hohenegger et al. 2006; Zhang et al. 2007; Selz and Craig 2015). A deeper understanding of the variance growth might be gained by spectral analyses, revealing the scales at which the energy of nondivergent and irrotational wind resides. Since moist convection plays a key role in creating the " $5 / 3$ slope" of the atmospheric energy spectrum (Durran and Weyn 2016; Sun et al. 2017), it would be highly interesting to investigate the properties of the spectra for the different cases and their variations with respect to the diurnal cycle of moist convection.

Acknowledgments. The authors thank Julian Quinting for providing the source code for the decomposition of the horizontal wind and for fruitful discussions. CK acknowledges funding from the CTI-Project 16124.1 PFEN-IW "Prévision hydrologique à haute performance pour la production hydroélectrique." This work was supported by the Swiss National Supercomputing Center (CSCS) under project ID s83. We also thank three anonymous reviewers for their constructive comments.

\section{REFERENCES}

Baldauf, M., A. Seifert, J. Förstner, D. Majewski, M. Raschendorfer, and T. Reinhardt, 2011: Operational convective-scale numerical weather prediction with the COSMO model: Description and sensitivities. Mon. Wea. Rev., 139, 3887-3905, https://doi.org/ 10.1175/MWR-D-10-05013.1.

Bei, N., and F. Zhang, 2007: Impacts of initial condition errors on mesoscale predictability of heavy precipitation along the MeiYu front of China. Quart. J. Roy. Meteor. Soc., 133, 83-99, https://doi.org/10.1002/qj.20.

Bierdel, L., T. Selz, and G. C. Craig, 2017: Theoretical aspects of upscale error growth through the mesoscales: An analytical model. Quart. J. Roy. Meteor. Soc., 143, 3048-3059, https://doi.org/ 10.1002/qj.3160.
Buizza, R., M. Miller, and T. N. Palmer, 1999: Stochastic representation of model uncertainties in the ECMWF Ensemble Prediction System. Quart. J. Roy. Meteor. Soc., 125, 28872908, https://doi.org/10.1002/qj.49712556006.

Done, J. M., G. C. Craig, S. L. Gray, P. A. Clark, and M. E. B. Gray, 2006: Mesoscale simulations of organized convection: Importance of convective equilibrium. Quart. J. Roy. Meteor. Soc., 132, 737-756, https://doi.org/10.1256/qj.04.84.

Durran, D. R., and M. Gingrich, 2014: Atmospheric predictability: Why butterflies are not of practical importance. J. Atmos. Sci., 71, 2476-2488, https://doi.org/10.1175/JAS-D-14-0007.1.

, and J. A. Weyn, 2016: Thunderstorms do not get butterflies. Bull. Amer. Meteor. Soc., 97, 237-243, https://doi.org/10.1175/ BAMS-D-15-00070.1.

Fresnay, S., A. Hally, C. Garnaud, E. Richard, and D. Lambert, 2012: Heavy precipitation events in the Mediterranean: Sensitivity to cloud physics parameterisation uncertainties. Nat. Hazards Earth Syst. Sci., 12, 2671-2688, https://doi.org/ 10.5194/nhess-12-2671-2012.

Fuhrer, O., C. Osuna, X. Lapillonne, T. Gysi, B. Cumming, M. Bianco, A. Arteaga, and T. Schulthess, 2014: Towards a performance portable, architecture agnostic implementation strategy for weather and climate models. Supercomput. Front. Innovations, 1, 45-62, https://doi.org/10.14529/jsfi140103.

Gebhardt, C., S. E. Theis, M. Paulat, and Z. Ben Bouallègue, 2011: Uncertainties in COSMO-DE precipitation forecasts introduced by model perturbations and variation of lateral boundaries. Atmos. Res., 100, 168-177, https://doi.org/10.1016/ j.atmosres.2010.12.008.

Hally, A., E. Richard, S. Fresnay, and D. Lambert, 2014: Ensemble simulations with perturbed physical parameterizations: PreHyMeX case studies. Quart. J. Roy. Meteor. Soc., 140, 19001916, https://doi.org/10.1002/qj.2257.

Hohenegger, C., and C. Schär, 2007: Predictability and error growth dynamics in cloud-resolving models. J. Atmos. Sci., 64, 4467-4478, https://doi.org/10.1175/2007JAS2143.1.

— D. Lüthi, and C. Schär, 2006: Predictability mysteries in cloud-resolving models. Mon. Wea. Rev., 134, 2095-2107, https://doi.org/10.1175/MWR3176.1.

Holton, J. R., and G. J. Hakim, 2013: An Introduction to Dynamic Meteorology. 5th ed. Academic Press, $552 \mathrm{pp}$.

Keil, C., F. Heinlein, and G. C. Craig, 2014: The convective adjustment time-scale as indicator of predictability of convective precipitation. Quart. J. Roy. Meteor. Soc., 140, 480-490, https://doi.org/10.1002/qj.2143.

Klasa, C., M. Arpagaus, A. Walser, and H. Wernli, 2018: An evaluation of the convection-permitting ensemble COSMO-E for three contrasting precipitation events in Switzerland. Quart. J. Roy. Meteor. Soc., 144, 744-764, https://doi.org/ 10.1002/qj.3245.

Kühnlein, C., C. Keil, G. C. Craig, and C. Gebhardt, 2014: The impact of downscaled initial condition perturbations on convective-scale ensemble forecasts of precipitation. Quart. J. Roy. Meteor. Soc., 140, 1552-1562, https://doi.org/10.1002/ qj.2238.

Lorenz, E. N., 1969: The predictability of a flow which possesses many scales of motion. Tellus, 21, 289-307, https://doi.org/ 10.3402/tellusa.v21i3.10086.

_ 1982: Atmospheric predictability experiments with a large numerical model. Tellus, 34, 505-513, https://doi.org/10.3402/ tellusa.v34i6.10836.

Luo, Y., and Y. Chen, 2015: Investigation of the predictability and physical mechanisms of an extreme-rainfall-producing mesoscale 
convective system along the Meiyu front in East China: An ensemble approach. J. Geophys. Res. Atmos., 120, 10 593-10618, https://doi.org/10.1002/2015JD023584.

Lynch, P., 1988: Deducing the wind from vorticity and divergence. Mon. Wea. Rev., 116, 86-93, https://doi.org/10.1175/1520-0493(1988) 116<0086:DTWFVA $>2.0 . \mathrm{CO} ; 2$.

— 1989: Partitioning the wind in a limited domain. Mon. Wea. Rev., 117, 1492-1500, https://doi.org/10.1175/1520-0493(1989) $117<1492$ :PTWIAL $>2.0$. CO;2.

Melhauser, C., and F. Zhang, 2012: Practical and intrinsic predictability of severe and convective weather at the mesoscales. J. Atmos. Sci., 69, 3350-3371, https://doi.org/10.1175/JAS-D-11-0315.1.

Murphy, J. M., 1988: The impact of ensemble forecasts on predictability. Quart. J. Roy. Meteor. Soc., 114, 463-493, https:// doi.org/10.1002/qj.49711448010.

Nielsen, E. R., and R. S. Schumacher, 2016: Using convectionallowing ensembles to understand the predictability of an extreme rainfall event. Mon. Wea. Rev., 144, 3651-3676, https://doi.org/10.1175/MWR-D-16-0083.1.

Press, W. H., B. P. Flannery, S. A. Teukolsky, and W. T. Vetterling, 1992: Numerical Recipes in Fortran 77: The Art of Scientific Computing. 2nd ed. Cambridge University Press, 580 pp.

Schubert, W. H., J. J. Hack, P. L. Silva Dias, and S. R. Fulton, 1980: Geostrophic adjustment in an axisymmetric vortex. J. Atmos. Sci., 37, 1464-1484, https://doi.org/10.1175/1520-0469(1980) 037<1464:GAIAAV >2.0.CO;2.

Selz, T., and G. Craig, 2015: Upscale error growth in a highresolution simulation of a summertime weather event over Europe. Mon. Wea. Rev., 143, 813-827, https://doi.org/10.1175/ MWR-D-14-00140.1.

Sun, Y. Q., and F. Zhang, 2016: Intrinsic versus practical limits of atmospheric predictability and the significance of the butterfly effect. J. Atmos. Sci., 73, 1419-1438, https://doi.org/10.1175/JAS-D-150142.1.

, R. Rotunno, and F. Zhang, 2017: Contributions of moist convection and internal gravity waves to building the atmospheric $-5 / 3$ kinetic energy spectra. J. Atmos. Sci., 74, 185-201, https://doi.org/10.1175/JAS-D-16-0097.1.
Tan, Z.-M., F. Zhang, R. Rotunno, and C. Snyder, 2004: Mesoscale predictability of moist baroclinic waves: Experiments with parameterized convection. J. Atmos. Sci., 61, 17941804, https://doi.org/10.1175/1520-0469(2004)061<1794: MPOMBW $>2.0 . \mathrm{CO} ; 2$.

Walser, A., D. Lüthi, and C. Schär, 2004: Predictability of precipitation in a cloud-resolving model. Mon. Wea. Rev., 132, 560-577, https://doi.org/10.1175/1520-0493(2004)132<0560: POPIAC $>2.0 . \mathrm{CO} ; 2$.

Weyn, J. A., and D. R. Durran, 2017: The dependence of the predictability of mesoscale convective systems on the horizontal scale and amplitude of initial errors in idealized simulations. J. Atmos. Sci., 74, 2191-2210, https://doi.org/10.1175/ JAS-D-17-0006.1.

Xu, Q., J. Cao, and S. Gao, 2011: Computing streamfunction and velocity potential in a limited domain of arbitrary shape. Part I: Theory and integral formulae. Adv. Atmos. Sci., 28, 14331444, https://doi.org/10.1007/s00376-011-0185-6.

Zhang, F., C. Snyder, and R. Rotunno, 2002: Mesoscale predictability of the "surprise" snowstorm of 24-25 January 2000. Mon. Wea. Rev., 130, 1617-1632, https://doi.org/10.1175/15200493(2002)130<1617:MPOTSS > 2.0.CO;2.

,-- , and — 2003: Effects of moist convection on mesoscale predictability. J. Atmos. Sci., 60, 1173-1185, https://doi.org/ 10.1175/1520-0469(2003)060<1173:EOMCOM>2.0.CO;2.

_ - A. M. Odins, and J. W. Nielsen-Gammon, 2006: Mesoscale predictability of an extreme warm-season precipitation event. Wea. Forecasting, 21, 149-166, https://doi.org/10.1175/ WAF909.1.

— N. Bei, R. Rotunno, C. Snyder, and C. C. Epifanio, 2007: Mesoscale predictability of moist baroclinic waves: Convection-permitting experiments and multistage error growth dynamics. J. Atmos. Sci., 64, 3579-3594, https://doi.org/ 10.1175/JAS4028.1.

Zimmer, M., G. C. Craig, C. Keil, and H. Wernli, 2011: Classification of precipitation events with a convective response timescale and their forecasting characteristics. Geophys. Res. Lett., 38, L05802, https://doi.org/10.1029/2010GL046199. 